\title{
Color-Related Signals in the Primate Superior Colliculus
}

\author{
Brian J. White, ${ }^{1}$ Susan E. Boehnke, ${ }^{1}$ Robert A. Marino, ${ }^{1}$ Laurent Itti, ${ }^{2}$ and Douglas P. Munoz ${ }^{1}$ \\ ${ }^{1}$ Centre for Neuroscience Studies, Queen's University, Kingston, Ontario K7L 3N6, Canada, and ${ }^{2}$ Department of Computer Science, University of Southern \\ California, Los Angeles, California 90089
}

\begin{abstract}
Color is important for segmenting objects from backgrounds, which can in turn facilitate visual search in complex scenes. However, brain areas involved in orienting the eyes toward colored stimuli in our environment are not believed to have access to color information. Here, we show that neurons in the intermediate layers of the monkey superior colliculus (SC), a critical structure for the production of saccadic eye movements, can respond to isoluminant color stimuli with the same magnitude as a maximum contrast luminance stimulus. In contrast, neurons from the superficial SC layers showed little color-related activity. Crucially, visual onset latencies were 30-35 ms longer for color, implying that luminance and chrominance information reach the SC through distinct pathways and that the observed colorrelated activity is not the result of residual luminance signals. Furthermore, these differences in visual onset latency translated directly into differences in saccadic reaction time. The results demonstrate that the saccadic system can signal the presence of chromatic stimuli only one stage from the brainstem premotor circuitry that drives the eyes.
\end{abstract}

\section{Introduction}

Human and nonhuman primates are the only mammals with trichromatic color vision (Jacobs, 1993), and it is generally believed that this system was driven by the nutritional advantage of discriminating ripe fruit from foliage (Mollon, 1989; Regan et al., 2001) and edible leaves (Dominy and Lucas, 2001). Color therefore plays an important role in segmenting objects from backgrounds, which can in turn facilitate visual search (D'Zmura et al., 1997) and the recognition of natural scenes (Gegenfurtner and Rieger, 2000). However, the neural representation of color is widely believed to be absent in brain areas that control saccadic eye movements (Marrocco and Li, 1977; Schiller and Malpeli, 1977; Schiller et al., 1979; Ottes et al., 1987), which serve to direct gaze toward colored objects in our environment. Instead, the saccadic system is thought to be essentially color-blind and predominantly driven by the achromatic "broadband" or luminance system (Schiller et al., 1979), which is relayed through the magnocellular layers of the lateral geniculate nucleus (LGN) to visual cortex. There is, however, a large network of direct and indirect projections from visual cortex (including areas involved in color processing) to saccadic substrates, in particular the superior colliculus (SC) (Fries, 1984; Lui et al., 1995; Lock et al., 2003), a critical structure in the control of overt and covert visual orienting (Kustov and Robinson, 1996; Ignashchenkova et al., 2004; Fecteau and Munoz, 2006; Dorris et al., 2007). Furthermore, behavioral observations are in sharp contrast with the assumption that the saccadic system is color-blind: human observers have no difficulty in detecting and rapidly orienting toward chromatic

Received April 27, 2009; revised July 24, 2009; accepted Aug. 24, 2009.

This work was supported by Human Frontiers Science Program Grant RGP0039-2005-Cand Canadian Institutes of Health Research Grants MOP-77734 and CNS-90910. D.P.M. was supported by the Canada Research Chair Program. We thank Ann Lablans, Becky Cranham, Donald Brien, Sean Hickman, and Mike Lewis for technical assistance.

Correspondence should be addressed to Brian J. White at the above address. E-mail: brianw@biomed. queensu.ca.

DOI:10.1523/JNEUROSCI.1986-09.2009

Copyright $\odot 2009$ Society for Neuroscience ～0270-6474/09/2912159-08\$15.00/0 stimuli that are isoluminant with the background (White et al., 2006), implying that some information about stimulus location is propagated to the saccadic system.

Here, we sought to test for a direct neural correlate of color information in the saccadic system using stimuli derived from the Derrington-Krauskopf-Lennie (DKL) color space (Krauskopf et al., 1982; Derrington et al., 1984), and visible primarily through a chromatic difference from the background (i.e., visible primarily to color-sensitive mechanisms). We report the first evidence that neurons in the primate SC can respond vigorously to such stimuli, and although they tend to show limited color specificity, these color-related signals appear to involve different pathways than luminance as evidenced by differences in visual onset latency, and the recruitment of primarily neurons with a sustained visual response from the intermediate SC layers.

\section{Materials and Methods}

Data were collected from two rhesus monkeys (Macaca mulatta; 11 and $12 \mathrm{~kg}$ ). Extracellular recording techniques as well as surgical procedures have been described previously (Marino et al., 2008).

Stimuli and equipment. Stimuli were presented on a cathode ray tube monitor at a screen resolution of $1024 \times 768$ pixels $(75 \mathrm{~Hz}$ noninterlaced), subtending a viewing angle of $54 \times 44^{\circ}$. The voltage-toluminance relationship (gamma) for each of the phosphors of the monitor was linearized using the UDT Instruments S471 optometer with a model 265 photopic filter. The color properties of the monitor were measured using the PR-655 (Photoresearch), and corrections were made for the Judd-Vos modified luminosity function (Judd, 1951; Vos, 1978). Stimulus presentation and data acquisition were controlled by a UNIXbased real-time data control system (REX) (Hays et al., 1982). Spikes, eye position data, and event data were sampled at $40 \mathrm{kHz}$ and recorded in a multichannel data acquisition system (Plexon).

Target stimuli were Gaussian windowed spots $\left(\mathrm{SD}, 0.7^{\circ}\right)$ whose color properties were derived from the DKL color space (Krauskopf et al., 1982; Derrington et al., 1984) (see Fig. 1b). This color space corresponds closely to the type of segregation that exists along the geniculostriate pathway in early vision (Derrington et al., 1984). One pathway sums the inputs of the long- and middle-wavelength cones $(\mathrm{L}+\mathrm{M})$, producing a 
luminance channel that is mostly sensitive to stimuli varying along the "black-white" dimension in the DKL space. A second pathway computes the difference between the inputs of the $\mathrm{L}$ and $\mathrm{M}$ cones $(\mathrm{L}-\mathrm{M})$, and is mostly sensitive to stimuli varying along the "red-green" dimension in the DKL space. A third pathway computes the difference between the inputs of the short-wavelength cones (S-cones) with the sum of the Land $\mathrm{M}$-cones $[\mathrm{S}-(\mathrm{L}+\mathrm{M})]$, and is mostly sensitive to stimuli varying along the "blue-yellow" dimension in the DKL space. These three channels form the primary visual-cortical inputs via the magnocellular, parvocellular, and koniocellular layers of the LGN, respectively.

The target color stimuli were chosen from $30^{\circ}$ steps around the azimuth in the isoluminant DKL color plane (illustrated by the small circles superimposed around the plane) (see Fig. $1 b$ ) and were presented against an isoluminant $\left(20.5 \mathrm{~cd} / \mathrm{m}^{2}\right)$ neutral gray background illustrated by the very center point of the DKL space. Background illumination was well above scotopic, rod-dominated, light levels (typically $<1 \mathrm{~cd} / \mathrm{m}^{2}$ ) (Hess et al., 1990). Post hoc measurements of the luminosity of each color stimulus showed negligible deviations $\left(<0.3 \mathrm{~cd} / \mathrm{m}^{2}\right.$ for all colors) from the background luminance (see supplemental Fig. $S 1 a$, available at www. jneurosci.org as supplemental material). The achromatic control stimulus was presented at $100 \%$ luminance contrast (black) and corresponds to the very most bottom point in the DKL space (see Fig. $1 b$ ). Along the cardinal color axes, the maximum achievable cone contrasts for our stimuli were $\sim 7 \%$ for $\mathrm{L}$-cones, $13 \%$ for $\mathrm{M}$-cones, and $87 \%$ for S-cones. It is important to note that the maximum achievable cone contrast in the $\mathrm{L}-\mathrm{M}$ (red-green) direction is naturally only approximately one-tenth of the cone contrast of the $\mathrm{L}+\mathrm{M}$ (black-white) direction because of the overlap in the spectral sensitivities of the L- and M-cones.

Procedure. Monkeys were seated in a primate chair (Crist Instrument), head-restrained facing the video monitor. Once an SC neuron was isolated, its visual response field was mapped using a rapid visual stimulation procedure. On a given trial, a rapid series of 14 stimuli were flashed (100 ms duration and $150 \mathrm{~ms}$ interstimulus intervals) at random locations across the visual field. There were 182 possible stimulus locations evenly dispersed across the visual field, and all locations were stimulated once after 13 consecutive trials. Spikes were collected over a small epoch from 60 to $90 \mathrm{~ms}$ after each stimulus onset and averaged on a trial-bytrial basis using a real-time mapping program written in Matlab (MathWorks). Activity was interpolated between stimulus locations to produce a smooth map denoting the "hot spot" of the neuron, usually within $\sim 40-50$ trials.

Once the visual response field was mapped, monkeys performed a delayed saccade task (see Fig. 1a). Each trial started with fixation of a black fixation spot for $500-800 \mathrm{~ms}$, and then a target stimulus appeared in the response field of the neuron. After a delay (500-800 ms), the fixation spot was removed and a saccade was generated toward the target stimulus for a liquid reward. The monkey first performed the task using the luminance-based target stimulus, and then using randomly selected DKL color stimuli (previously described).

For the behavioral "gap" task, the fixation point was removed $200 \mathrm{~ms}$ before the onset of the target stimulus to facilitate the disengagement of visual fixation while measuring the visual triggering capability of the isoluminant color stimuli relative to the maximum contrast luminance stimulus. Here, isoluminant color stimuli were chosen from the cardinal color axes only (red, green, blue, and yellow). Stimuli were presented randomly left or right of fixation at $12^{\circ}$ eccentricity.

Analyses and neuron classification. Individual spikes were sorted offline using Plexon Off-line Sorting software (Plexon) to remove artifacts and verify single units. Spike density functions were created by convolving individual spikes with a function that resembles a postsynaptic potential (Thompson et al., 1996) described by the following equation: $R(t)=\left(1-\exp \left(-t / \tau_{g}\right)\right) \times\left(\exp \left(-t / \tau_{d}\right)\right)$, where firing rate $R$, as a function of time $t$, varies according a time constant for a growth phase $\tau_{g}$, and a time constant for a decay phase $\tau_{d}$. Consistent with Thompson et al. (1996), we chose time constants of 1 and 20 for the growth and decay phases, respectively.

SC neurons have fairly characteristic responses ranging from pure visual to pure motor and have been well described (Mays and Sparks, 1980; Munoz and Wurtz, 1995; McPeek and Keller, 2002). The terms "phasic" versus "tonic" have been used to describe the visual characteristics of SC neurons (McPeek and Keller, 2002; Li and Basso, 2008), phasic referring to a short visual burst and tonic referring to an initial visual burst followed by an extended period of lower frequency activity. SC neurons are often categorized as one or the other. However, in the intermediate layers, visual responses more often fall along a continuum between these two extremes. In the current study, stimuli were designed to target the luminance or color-opponent divisions of the geniculostriate pathway in early vision, whose neurons characteristically show a "transient" versus "sustained" visual response profile, respectively (Kaplan et al., 1990). We therefore adopted this terminology for SC visual responses in the current paper.

Note that what we refer to as "sustained-visual" SC neurons correspond to what has been termed "tonic-visual" neurons by others (McPeek and Keller, 2002; Li and Basso, 2008). These are also thought to be similar to the so-called "quasivisual" neurons first described by Mays and Sparks (1980). However, the double-saccade test was not performed to confirm this. Our "sustained-visuomotor" neurons likely correspond to "visuo-movement prelude" neurons (McPeek and Keller, 2002) and "build-up" neurons (Munoz and Wurtz, 1995; Dorris and Munoz, 1998), but we did not formally test for prelude or build-up activity.

We derived a transient index similar to that of Schiller and Malpeli (1977) to classify neurons as either visually transient or sustained using the following equation: Transient index $=(I /(I+D)) \times 100$, which defines a ratio between $I$, the maximum activity within an initial period of the visual response, and $D$, the average activity during a delay period of the visual response (note that classifications were made using the maximum contrast luminance stimulus). The initial period was chosen between 50 and $100 \mathrm{~ms}$ from target onset because most peak responses for our luminance stimulus occurred within this range. The delay epoch started where the initial epoch ended, extending from 100 to $500 \mathrm{~ms}$ after target onset. The main advantage of the large delay epoch was to insure that we obtained a reliable sample of the sustained profile while avoiding sparse periods of inactivity that are not reflective of the overall sustained profile of the neuron. Transient indices could range from 50 to 100 , and neurons with an index $\geq 95$ were considered transient, but otherwise were considered sustained. We chose a transient criterion of 95 to bias against misclassifying neurons as transient unless they were very transient.

Neurons from the superficial SC are invariably visual (Marrocco and $\mathrm{Li}, 1977$ ) and correspond to the "transient-visual" type described here. They were clearly sampled from the uppermost region of the superficial SC so we can state with confidence that they were located in the superficial SC. Our sample of sustained-visual neurons were sampled well below this (typically deeper than $1 \mathrm{~mm}$ ) and appear to correspond to the tonicvisual neurons described by McPeek and Keller (2002), which are believed to be located around the junction between the superficial and intermediate SC. Thus, it is not absolutely clear whether they belong to the lower region of the superficial SC or the upper region of the intermediate SC. Visuomotor neurons with transient or sustained visual responses were invariably found below both transient- and sustained-visual neurons. A neuron was defined as visuomotor if the average firing rate right around the time of saccade onset $(-25$ to $+25 \mathrm{~ms}$ ) was $>3$ SDs above a presaccadic epoch (defined as the average firing rate from -150 to $-50 \mathrm{~ms}$ before saccade onset).

A color sensitivity index, described by Johnson et al. (2008), was computed for correlations with the visual transient index. This was simply a ratio of the peak response for the optimal color over the peak response for our maximum luminance contrast stimulus: Color sensitivity index $=$ peak (optimal color)/peak (100\% lum contrast).

It is important to note that this color sensitivity index will vary dramatically depending on the contrast used to measure the responses to isoluminant versus luminant stimuli. Therefore, indices obtained here are not directly comparable with those obtained in other studies.

For statistical analyses, visual onset latencies were defined as the time relative to target stimulus onset in which visual activity first exceeded 3 SDs above a baseline (defined as the average activity of a $100 \mathrm{~ms}$ epoch before target stimulus onset) and remained so for a reasonable period of time (>35 ms). Peak visual response was the maximum response within 


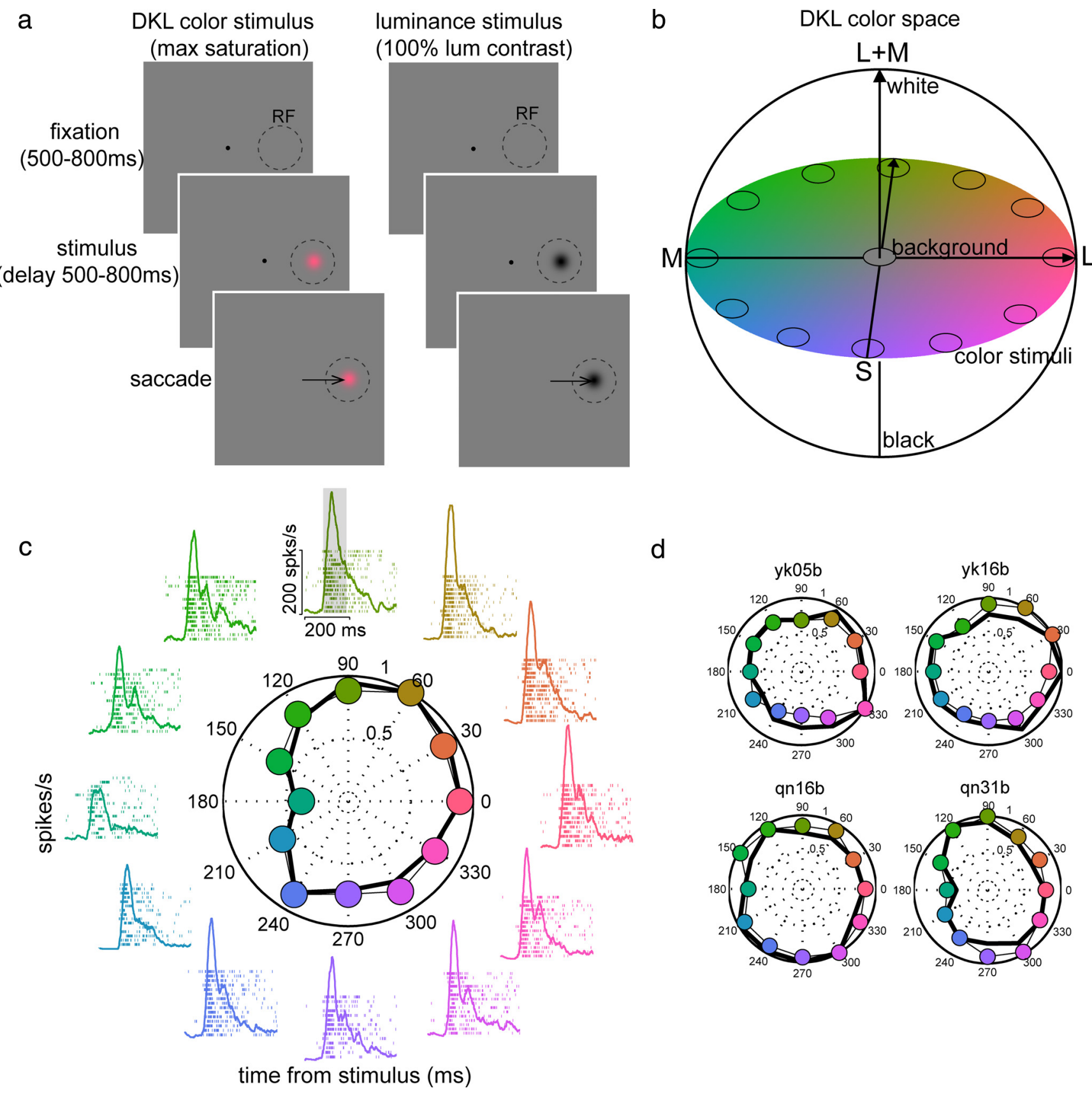

Figure 1. $\boldsymbol{a}$, Delayed saccade task. The monkey fixated a central black spot for $500-800 \mathrm{~ms}$, and then a randomly chosen isoluminant color stimulus (Gaussian windowed; $S D, 0.7^{\circ}$ ) appeared in the response field (RF) of the neuron. After a delay ( $500-800 \mathrm{~ms}$ ), the fixation spot was removed, serving as a go-signal for a saccade toward the color stimulus. Comparisons were made with an achromatic black stimulus presented at 100\% luminance contrast. $\boldsymbol{b}$, DKL color space (Krauskopf et al., 1982; Derrington et al., 1984) (see Materials and Methods). Color stimuli were chosen from $30^{\circ}$ steps around the azimuth of the isoluminant plane at maximum saturation (illustrated by the circles superimposed on the color plane). The stimuli were presented against an isoluminant neutral gray background $\left(20.5 \mathrm{~cd} / \mathrm{m}^{2}\right)$ illustrated by the center point in the color plane. The achromatic black control stimulus corresponds to the very most bottom point in this space. $c$, Rasters and spike density functions of a single sustained-visual SC neuron to each of the color stimuli (aligned on stimulus onset). At the center is a polar plot of the normalized peak responses for each color (colored symbols linked by the thin line). The thicker line shows the responses averaged over a short epoch ( $80-180 \mathrm{~ms}$ ) defined by the gray shaded region. $\boldsymbol{d}$, Color tuning profiles for four additional sustained-visual neurons.

$150 \mathrm{~ms}$ of target onset. For statistical comparisons, delay activity was defined as the average activity from 150 to $500 \mathrm{~ms}$ after target stimulus onset for our maximum contrast luminance stimuli, and 180 to 530 for our isoluminant color stimuli. The slight difference in the timing of these epochs was to account for the color-related delay in visual onset latency (see Fig. 2).

\section{Results}

We recorded the activity of 68 visually responsive neurons from the superficial (within $1 \mathrm{~mm}$ of SC surface) and intermediate layers (1-3 mm from SC surface) of the SC in two rhesus monkeys (Macaca mulatta) trained to perform a delayed saccade task using color stimuli presented against an isoluminant background (Fig. 1a) (for details, see Materials and Methods).

Neurons were categorized into two general visual classes (transient vs sustained) and whether or not they had motor activity (see Materials and Methods). Figure $1 c$ shows rasters and spike density functions for a single sustained-visual neuron to the various colors chosen from $30^{\circ}$ steps around the azimuth in the 
DKL color plane (Fig. 1b). At the center is a polar plot showing the normalized peak response of the neuron for each color. This neuron showed a remarkably large response (161-432 spikes/s) to all isoluminant colors with a fairly broad color preference. Most of our sustained-visual/ visuomotor neurons showed similarly strong responses (Fig. 1d), 36\% (21 of 59) of which were moderately, although significantly, tuned $\left[p<0.01, r^{2}\right.$ from 0.65 to 0.93, planar-fit regression-based test for directional tuning (Kurtzer et al., 2005)], indicating the presence of at least some broad color preferences.

A critical question is whether these are true color-related signals (i.e., signals originating from the color-opponent system in early vision) or simply residual luminance signals. Residual luminance could render a photometrically isoluminant stimulus detectable from its background via luminance-sensitive mechanisms. This may arise from (1) less than perfect stimulus calibration, (2) variation in individual observer's isoluminant point, or (3) variation in the isoluminance balance of the individual neuron (Logothetis et al., 1990; Dobkins and Albright, 1994; Gegenfurtner et al., 1994) (see supplemental data, available at www.jneurosci.org as supplemental material). We would nonetheless expect the effect of residual luminance to be relatively small, at least compared with the magnitude of the responses observed here [e.g., somewhere in the range of $25 \%$ of the maximum response (Gegenfurtner et al., 1994)]. Given the size of the observed color-related response, we took a conservative approach to this issue by making comparisons between the optimal color of each neuron and an achromatic stimulus of $100 \%$ luminance contrast (Fig. 1a). This represents the maximum degree of separation possible between test and control stimuli within this color space, such that if residual luminance is responsible for the observed response to our color stimuli, it should be at least smaller than the response to an achromatic stimulus of maximum luminance contrast.

Figure 2 shows the average visual responses (normalized to the maximum) for the four cell types. The top two panels show average responses for the maximum contrast luminance stimulus (black traces) and the optimal color stimulus (colored traces) for our sample of sustained-visual (Fig. 2a) and transient-visual (Fig. $2 b$ ) neurons. The bottom two panels (Fig. $2 c, d$ ) show average responses for visuomotor neurons with sustained and transient visual responses. For our sample of sustained-visual (Fig. $2 a$ ) and sustained-visuomotor (Fig. 2c) neurons, the average optimal color-related response (red traces) was remarkably high compared with luminance (black traces), with approximately equal peak activity (sustained-visual, $t_{(18)}=1.43, p>0.1$; sustainedvisuomotor, $\left.t_{(25)}=-0.02, p>0.9\right)$ and even slightly greater delay activity for color (sustained-visual, $t_{(18)}=3.24, p=0.005$; sustained-visuomotor, $t_{(25)}=3.17, p=0.004$ ) (for derivations of peak and delay activity and visual onset latency, see Materials and Methods). However, visual onset latencies were on average 25-30
Sustained
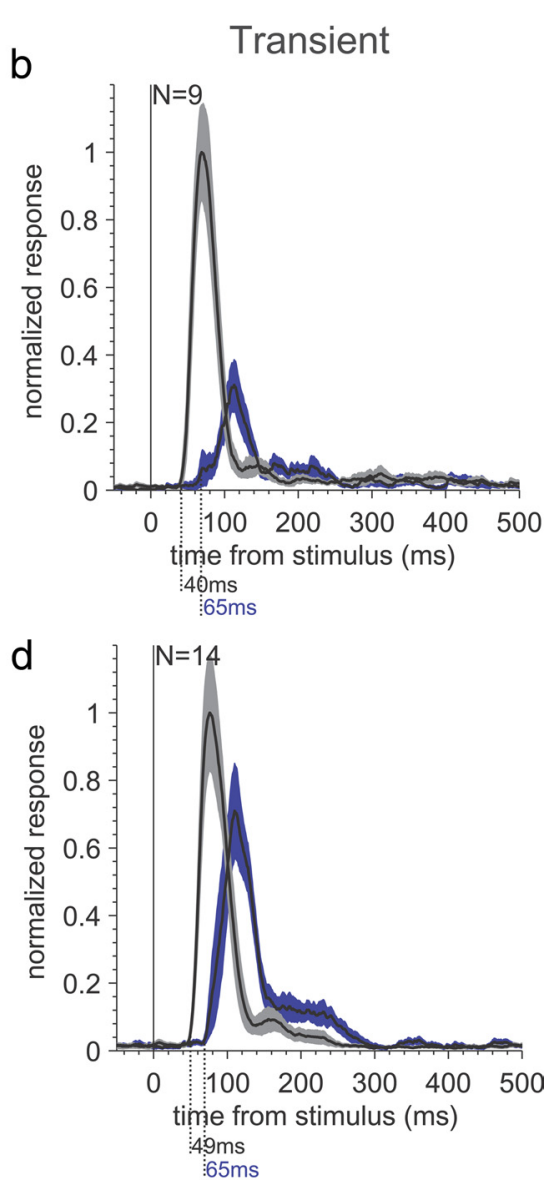

Figure 2. Comparison of the visual responses for the optimal color stimuli (colored traces) and maximum contrast luminance stimulus (black traces) across SC cell types. The top two panels show average normalized spike density functions for sustainedvisual neurons to their optimal color stimulus (red traces) and the maximum contrast luminance stimulus (black traces) (a), and b). $\boldsymbol{c}$ and $\boldsymbol{d}$ show averages for our sample of visuomotor neurons for the same two visual classifications. Note that transient-visual neurons $(\boldsymbol{b})$ originated from the superficial SC layers ( $<1 \mathrm{~mm}$ from SC surface). Below each panel is the visual onset latency for each response profile. The shaded regions represent \pm 1 SEM.

ms longer for color for these two classes of neurons (sustainedvisual, $t_{(18)}=6.25, p<0.00001$; sustained-visuomotor, $t_{(25)}=$ $4.48, p<0.001)$. In contrast, for our sample of transient-visual neurons (Fig. $2 b$ ), the best color-related response (blue traces) was reduced to approximately one-quarter of the magnitude of the $100 \%$ contrast luminance response $\left(t_{(8)}=-5.75 ; p<0.001\right)$. This result is consistent with previous reports that the superficial SC layers do not receive color-opponent inputs (Marrocco and Li, 1977; Schiller and Malpeli, 1977). The size of the color-related response for transient-visual neurons (Fig. $2 b$, blue traces) is also in close agreement with the degree of residual luminance expected from variations in the isoluminant balance of the individual neuron [e.g., as reported in the magnocellular division of the LGN (Logothetis et al., 1990), and middle temporal area (MT) (Dobkins and Albright, 1994; Gegenfurtner et al., 1994), which projects directly to the superficial SC layers (Lock et al., 2003)]. Visuomotor neurons with a transient visual response (Fig. 2d), which are located below the superficial SC layers, also showed fairly strong responses to color (blue traces). Key to all of these observations is that, if the color-related response seen in the SC was simply attributable to residual luminance, we would expect at best a weak response (at least smaller than our maximum contrast luminance stimulus) (Logothetis et al., 1990; Dobkins and Albright, 1994; Gegenfurtner et al., 1994), accompanied by a delay 
a
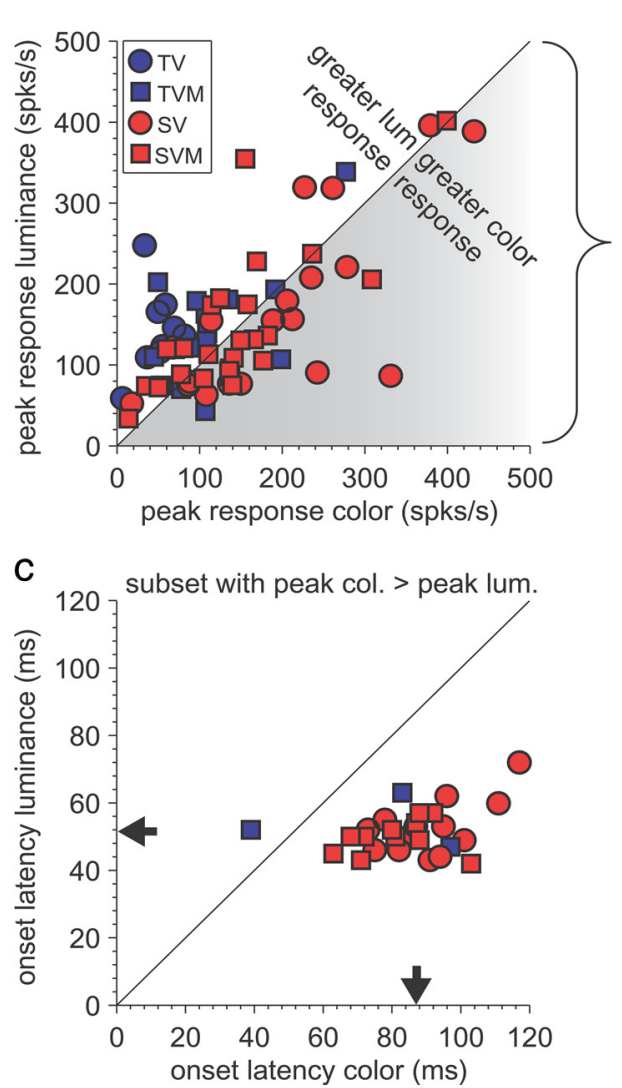

b

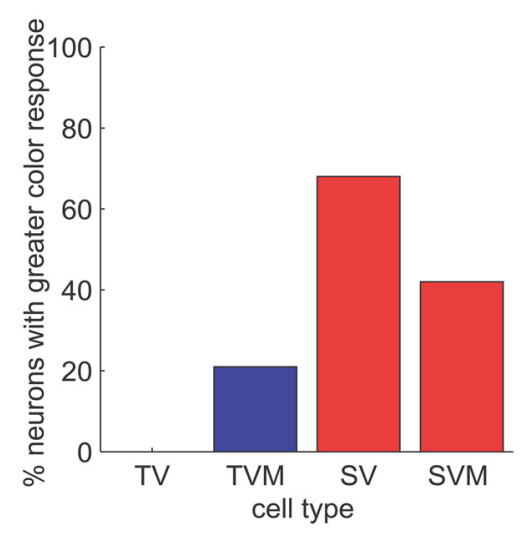

d

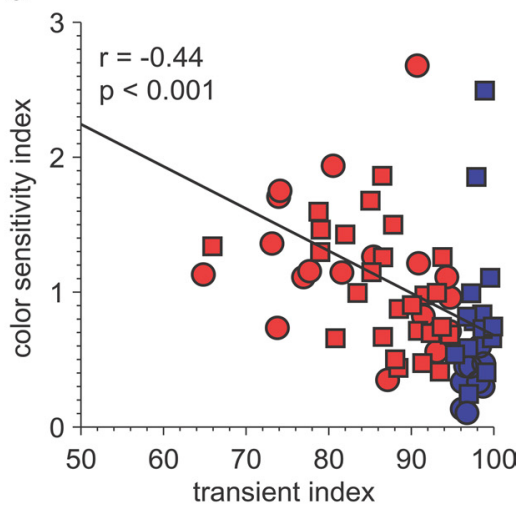

luminance stimuli (these data were not available from the delayed saccade task because saccades were not triggered by the appearance of the target stimulus). The stimuli were randomly interleaved and appeared $12^{\circ}$ left or right of fixation. Figure 4 confirms that the observed visual onset latency differences between luminance and color (medians of 36 and $38 \mathrm{~ms}$ collapsed across the cardinal DKL colors for monkeys $\mathrm{Y}$ and $\mathrm{Q}$, respectively) translated very closely into SRT differences (medians of 32 and $38 \mathrm{~ms}$ for monkeys $\mathrm{Y}$ and Q, respectively). That is, SRTs were mostly determined by the relative arrival times of these stimuli, even with relation to differences between colors (see supplemental data, available at www.jneurosci.org as supplemental material). This is also remarkably close to human SRT differences between luminance and color using DKL-derived stimuli [36 ms for stimuli collapsed across various eccentricities and colors (White et al., 2006)].

\section{Discussion}

These results provide strong evidence that the primate SC has access to visual signals originating in not only magnocellular (Schiller et al., 1979) but also the coloropponent divisions of the geniculostriate pathway. This implies that the saccadic system has the capability of signaling the presence of chromatic stimuli, which is in agreement with the fact that human observers have no difficulty in orienting toward such stimuli overtly (White et al., 2006). However, the broad color-tuning Summary of the peak visual responses and visual onset latencies for our sample of 68 neurons. $\boldsymbol{a}$, Peak visual responses. $\boldsymbol{b}$, Percentage neurons with greater color response. $\boldsymbol{c}$, Visual onset latencies. $\boldsymbol{d}$, A correlation between the color sensitivity index (Johnson et al., 2008) and transient index (Schiller and Malpeli, 1977) of each neuron (see Materials and Methods). TV, Transient-visual; TVM, transient-visuomotor; SV, sustained-visual; SVM, sustained-visuomotor. $\boldsymbol{b}$ and c represent a subset of the total sample of neurons whose peak visual response was greater for the optimal color than the maximum-contrast luminance stimulus. The arrows on the axes in c denote the median visual onset latency for the sample of neurons for color versus luminance.

in onset latency (Bell et al., 2006; Li and Basso, 2008). This is in sharp contrast to our observation of a strong response, yet accompanied by a significant delay in onset latency, particularly in terms of sustained-visual/visuomotor neurons (Fig. 2a,c; supplemental Fig. S1e,f, available at www.jneurosci.org as supplemental material).

Figure 3 summarizes the peak responses and visual onset latencies between color and luminance for each neuron across the cell types. A substantial percentage (41\% or 28 of 68 ) of the total sample responded better to their optimal color than the maximum contrast luminance stimulus (Fig. $3 a$, shaded region), and Figure $3 b$ shows the percentages across cell types. If we consider only those neurons with the better color-related response, the majority of these show longer visual onset latencies to color (Fig. $3 c$ ) and tend to have a sustained visual response profile (red symbols). In fact, there was a significant negative correlation between color sensitivity and the transient index of the neuron (Fig. $3 d$ ) $(r=-0.44 ; p<0.001)$ (for derivations of color sensitivity and transient indices, see Materials and Methods), indicating that neurons most sensitive to color tended to be the least transient (i.e., those with a more sustained response profile).

These differences in visual onset latency between color and luminance in the SC should correspond closely to differences in saccadic reaction time (SRT). To test this hypothesis, we had monkeys perform a gap saccade task (200 ms gap duration) (see Materials and Methods) to compare SRTs between color and profiles (Fig. $1 c, d$ ) suggest that information about color specificity may be limited in the SC. That is, these neurons appear to show strong "sensitivity," but only moderate "selectivity," for color. This almost certainly means that they receive inputs from multiple neurons tuned to different directions in color space (Sato et al., 1994).

The color-related responses we describe are unlikely because of residual luminance for several reasons: (1) The optimal colorrelated responses were often equal to or greater than maximum contrast luminance responses (Fig. 3). Even if our color stimuli carried a small residual luminance signal, they should have been at best only moderately "visible" to the SC if it were in fact colorblind. The magnitude of the color-related responses are especially remarkable given that the maximum achievable cone contrast for isoluminant stimuli (along the L-M direction) is naturally constrained to approximately one-tenth of the maximum cone contrast for luminance (see Materials and Methods). (2) The color-related responses were delayed by $\sim 30-35$ ms relative to luminance, independent of response magnitude. The most likely explanation for the delay is that the color-related signals traversed different pathways than luminance before finally converging on SC neurons. In other words, our chromatic stimuli were not simply acting like low-contrast luminance stimuli, as would be predicted if our results were primarily attributable to residual luminance (see supplemental data and Fig. S1e,f, available at www.jneurosci.org 
as supplemental material). A delayed color response without a corresponding attenuated peak response implies that these signals involve intrinsically different mechanisms than luminance. This is consistent with the idea that the observed color-related response in the $\mathrm{SC}$ is the result of visual signals originating from the color-opponent divisions of the geniculostriate pathway. However, this latency difference vastly exceeds the conduction time differences through LGN (Maunsell et al., 1999), suggesting a transcortical contribution as well. Furthermore, the observed differences in visual onset latency between color and luminance coincided closely with differences in SRT (Fig. 4 ), which were remarkably close to what has been previously reported in humans using similar DKL-derived stimuli (White et al., 2006). Finally, (3) the color-related activity was associated with neurons from the intermediate SC layers (Figs. 2, 3). The same was not true for transient-visual neurons from the superficial SC layers, which is consistent with previous reports (Marrocco and Li, 1977; Schiller and Malpeli, 1977).

We also confirmed that the colorrelated SC activity is indeed mostly visual and is unlikely because of activity related to motor planning or target selection (see supplemental data, available at www. jneurosci.org as supplemental material). This is particularly true for our sample of sustained visual neurons $(N=19)$, which showed the most robust color-related responses (Fig. $2 a$ ) and, by definition, do not have motor-related activity (see supplemental data and Fig. S2a, available at www.jneurosci.org as supplemental material) and do not typically show target selection activity (McPeek and Keller, 2002).

These results appear to be at odds with a previous study by Schiller et al. (1979), who examined the composition of the geniculostriate input into the SC by reversibly inactivating either the magnocellular (luminance) or parvocellular (color) layers of the LGN of anesthetized monkeys. They reported that the inactivation of the magnocellular layers eliminated visual activity in the SC, whereas the inactivation of the parvocellular layers did not. The authors concluded that the SC must be driven primarily by the achromatic broadband system. The fact that we do see a robust response to chromatic stimuli in the SC in awake monkeys implies that color-related activity in the SC might rely on the active engagement of the animal toward behaviorally relevant stimuli (Toth and Assad, 2002). For example, although the overt visual orienting system may have direct, bottom-up access to luminance, color may instead be transmitted to the SC via the same pathways that carry top-down, goal-related signals [e.g., via prefrontal cortex (Schall et al., 1995)]. The neurons carrying such signals might be either disrupted directly by the anesthesia itself or might only be active when awake animals perform goal-related tasks.

How might the SC gain access to color information? Dominant theories of perception and action have argued that visual pathways carrying signals for motor control ("dorsal stream") are mostly separate from visual pathways driving perception ("ventral stream") (Ungerleider and Mishkin, 1982; Goodale and Milner, 1992). Color was thought to belong exclusively to the latter (Livingstone and Hubel, 1988), but unlike the segregation that exists early in visual processing, color and luminance signals are not strictly segregated in the cortex (Sincich and Horton, 2005). Furthermore, the saccadic system receives projections from cortical areas belonging to both streams (Schall et al., 1995; Lock et al., 2003). The fact that we see such a dramatic difference between the response of transient-visual and sustained-visual/ visuomotor neurons to color is consistent with the different visual inputs into the superficial versus intermediate SC layers (Lock et al., 2003). Incidentally, neurons from the parvocellular (color) layers of the LGN are characteristically sustained and tend to have slightly longer visual onset latencies than neurons from the magnocellular (luminance) layers of the LGN, which are characteristically transient (Kaplan et al., 1990). At least some of the difference we see in visual onset latency between color and luminance may be attributable to differences originating from these early visual pathways (15-20 ms) (Schmolesky et al., 1998; Maunsell et al., 1999; McAlonan et al., 2008), but the remaining difference must arise elsewhere (i.e., transcortically).

The distribution of visual corticotectal projections is extensive (Fries, 1984; Lui et al., 1995; Lock et al., 2003), so it is difficult to determine the precise nature of the inputs driving the differences seen here. In addition to the direct retinotectal input, the superficial SC layers receive direct projections from visual areas V1, V2, 
V3, and MT (Fries, 1984; Lock et al., 2003), but the signals from these areas are not believed to be color-opponent (Marrocco and Li, 1977; Schiller and Malpeli, 1977; Schiller et al., 1979; Lia and Olavarria, 1996; Abel et al., 1997), and the results from our sample of transient-visual neurons support this (Fig. $2 b$ ). In contrast, the cortical projections to the intermediate SC layers are more complex, with direct inputs from frontal cortex (Leichnetz and Goldberg, 1988), plus extrastriate visual areas, some of which are traditionally associated with the ventral stream (Fries, 1984; Lock et al., 2003) (e.g., color-sensitive area V4). We do not yet have evidence that V4 neurons that project to the SC (Fries, 1984; Lock et al., 2003) are color-sensitive/selective, but given the colorrelated delay observed in the SC, this relatively later route (Schmolesky et al., 1998) is a possibility. Alternatively, the colorrelated signals might arise via an indirect route from $\mathrm{V} 4$ through the frontal eye field to the SC (Schall et al., 1995).

Our results also have important implications for the role of cone-specific signals in visual orienting. For example, Sumner et al. $(2002,2004)$ reported that stimuli invisible to the retinotectal and magnocellular pathways (S-cone isolating stimuli) can produce covert (as measured with manual reaction times), but not overt (as measured with saccades) orienting effects commonly associated with luminance-based stimuli [e.g., inhibition of return (Sumner et al., 2004)]. This was based on the premise that the superficial SC layers in particular do not receive inputs from S-cones (Marrocco and Li, 1977; Schiller and Malpeli, 1977), but until now it was not known whether neurons in the intermediate SC layers respond to color. In fact, $\sim 14 \%$ ( 8 of 59 ) of the colorresponsive neurons showed equal or greater responses in the S-cone direction than the maximum contrast luminance stimulus. Although we did not rigorously determine the optimal S-cone direction like Sumner et al. (2002, 2004), one would nonetheless predict at least some evidence of reduced visual activity around the S-cone direction if there were no such signals present in the SC. This was not the case (see supplemental Fig. S1b, available at www.jneurosci.org as supplemental material).

In sum, intermediate layer SC neurons are highly sensitive to isoluminant-color stimuli, and the color-related delay provides strong evidence that these signals traverse different pathways than luminance before converging on SC neurons. In natural visual environments, objects and the borders that define them are often isoluminant with the background (Hansen and Gegenfurtner, 2009). The intrinsic circuitry of the SC itself may use this information to make a better decision about saccade targeting.

\section{References}

Abel PL, O’Brien BJ, Lia B, Olavarria JF (1997) Distribution of neurons projecting to the superior colliculus correlates with thick cytochrome oxidase stripes in macaque visual area V2. J Comp Neurol 377:313-323.

Bell AH, Meredith MA, Van Opstal AJ, Munoz DP (2006) Stimulus intensity modifies saccadic reaction time and visual response latency in the superior colliculus. Exp Brain Res 174:53-59.

Derrington AM, Krauskopf J, Lennie P (1984) Chromatic mechanisms in lateral geniculate nucleus of macaque. J Physiol 357:241-265.

Dobkins KR, Albright TD (1994) What happens if it changes color when it moves?: the nature of chromatic input to macaque visual area MT. J Neurosci 14:4854-4870.

Dominy NJ, Lucas PW (2001) Ecological importance of trichromatic vision to primates. Nature 410:363-366.

Dorris MC, Munoz DP (1998) Saccadic probability influences motor preparation signals and time to saccadic initiation. J Neurosci 18:7015-7026.

Dorris MC, Olivier E, Munoz DP (2007) Competitive integration of visual and preparatory signals in the superior colliculus during saccadic programming. J Neurosci 27:5053-5062.

D'Zmura M, Lennie P, Tiana C (1997) Color search and visual field segregation. Percept Psychophys 59:381-388.
Fecteau JH, Munoz DP (2006) Salience, relevance, and firing: a priority map for target selection. Trends Cogn Sci 10:382-390.

Fries W (1984) Cortical projections to the superior colliculus in the macaque monkey: a retrograde study using horseradish peroxidase. J Comp Neurol 230:55-76.

Gegenfurtner KR, Rieger J (2000) Sensory and cognitive contributions of color to the recognition of natural scenes. Curr Biol 10:805-808.

Gegenfurtner KR, Kiper DC, Beusmans JM, Carandini M, Zaidi Q, Movshon JA (1994) Chromatic properties of neurons in macaque MT. Vis Neurosci 11:455-466.

Goodale MA, Milner AD (1992) Separate visual pathways for perception and action. Trends Neurosci 15:20-25.

Hansen T, Gegenfurtner KR (2009) Independence of color and luminance edges in natural scenes. Vis Neurosci 26:35-49.

Hays AV, Richmond BJ, Optican LM (1982) A UNIX-based multiple process system for real-time data acquisition and control. WESCON Conf Proc 2:1-10.

Hess R, Sharp L, Nordby K (1990) Night vision. Basic, clinical and applied aspects. Cambridge, UK: Cambridge UP.

Ignashchenkova A, Dicke PW, Haarmeier T, Thier P (2004) Neuronspecific contribution of the superior colliculus to overt and covert shifts of attention. Nat Neurosci 7:56-64.

Jacobs GH (1993) The distribution and nature of colour vision among the mammals. Biol Rev Camb Philos Soc 68:413-471.

Johnson EN, Hawken MJ, Shapley R (2008) The orientation selectivity of color-responsive neurons in macaque V1. J Neurosci 28:8096-8106.

Judd DB (1951) Report of U.S. Secretariat Committee on Colorimetry and Artificial Daylight. In: Twelfth Session of the CIE, p. 11. Stockholm, Paris: Bureau Central de la CIE.

Kaplan E, Lee BB, Shapley R (1990) New views of primate retinal function. In: Progress in retinal research (Osborne N, Chader G, eds), pp 273-336. New York: Pergamon, Oxford.

Krauskopf J, Williams DR, Heeley DW (1982) Cardinal directions of color space. Vision Res 22:1123-1131.

Kurtzer I, Herter TM, Scott SH (2005) Random change in cortical load representation suggests distinct control of posture and movement. Nat Neurosci 8:498-504.

Kustov AA, Robinson DL (1996) Shared neural control of attentional shifts and eye movements. Nature 384:74-77.

Leichnetz GR, Goldberg ME (1988) Reviews of oculomotor research. Neuroanatomy of the oculomotor system, pp 365-429. Amsterdam: Elsevier.

Li X, Basso MA (2008) Preparing to move increases the sensitivity of superior colliculus neurons. J Neurosci 28:4561-4577.

Lia B, Olavarria JF (1996) The distribution of corticotectal projection neurons correlates with the interblob compartment in macaque striate cortex. Vis Neurosci 13:461-466.

Livingstone M, Hubel D (1988) Segregation of form, color, movement, and depth: anatomy, physiology, and perception. Science 240:740-749.

Lock TM, Baizer JS, Bender DB (2003) Distribution of corticotectal cells in macaque. Exp Brain Res 151:455-470.

Logothetis NK, Schiller PH, Charles ER, Hurlbert AC (1990) Perceptual deficits and the activity of the color-opponent and broad-band pathways at isoluminance. Science 247:214-217.

Lui F, Gregory KM, Blanks RH, Giolli RA (1995) Projections from visual areas of the cerebral cortex to pretectal nuclear complex, terminal accessory optic nuclei, and superior colliculus in macaque monkey. J Comp Neurol 363:439-460.

Marino RA, Rodgers CK, Levy R, Munoz DP (2008) Spatial relationships of visuomotor transformations in the superior colliculus map. J Neurophysiol 100:2564-2576.

Marrocco RT, Li RH (1977) Monkey superior colliculus: properties of single cells and their afferent inputs. J Neurophysiol 40:844-860.

Maunsell JH, Ghose GM, Assad JA, McAdams CJ, Boudreau CE, Noerager BD (1999) Visual response latencies of magnocellular and parvocellular LGN neurons in macaque monkeys. Vis Neurosci 16:1-14.

Mays LE, Sparks DL (1980) Dissociation of visual and saccade-related responses in superior colliculus neurons. J Neurophysiol 43:207-232.

McAlonan K, Cavanaugh J, Wurtz RH (2008) Guarding the gateway to cortex with attention in visual thalamus. Nature 456:391-394.

McPeek RM, Keller EL (2002) Saccade target selection in the superior colliculus during a visual search task. J Neurophysiol 88:2019-2034. 
Mollon JD (1989) “Tho' she kneel'd in that place where they grew.” The uses and origins of primate colour vision. J Exp Biol 146:21-38.

Munoz DP, Wurtz RH (1995) Saccade-related activity in monkey superior colliculus. I. Characteristics of burst and buildup cells. J Neurophysiol 73:2313-2333.

Ottes FP, Van Gisbergen JA, Eggermont JJ (1987) Collicular involvement in a saccadic colour discrimination task. Exp Brain Res 66:465-478.

Regan BC, Julliot C, Simmen B, Viénot F, Charles-Dominique P, Mollon JD (2001) Fruits, foliage and the evolution of primate colour vision. Philos Trans R Soc Lond B Biol Sci 356:229-283.

Sato H, Katsuyama N, Tamura H, Hata Y, Tsumoto T (1994) Broad-tuned chromatic inputs to color-selective neurons in the monkey visual cortex. J Neurophysiol 72:163-168.

Schall JD, Morel A, King DJ, Bullier J (1995) Topography of visual cortex connections with frontal eye field in macaque: convergence and segregation of processing streams. J Neurosci 15:4464-4487.

Schiller PH, Malpeli JG (1977) Properties and tectal projections of monkey retinal ganglion cells. J Neurophysiol 40:428-445.

Schiller PH, Malpeli JG, Schein SJ (1979) Composition of geniculostriate input to superior colliculus of the rhesus monkey. J Neurophysiol 42:1124-1133.

Schmolesky MT, Wang Y, Hanes DP, Thompson KG, Leutgeb S, Schall JD,
Leventhal AG (1998) Signal timing across the macaque visual system. J Neurophysiol 79:3272-3278.

Sincich LC, Horton JC (2005) The circuitry of V1 and V2: integration of color, form, and motion. Annu Rev Neurosci 28:303-326.

Sumner P, Adamjee T, Mollon JD (2002) Signals invisible to the collicular and magnocellular pathways can capture visual attention. Curr Biol 12:1312-1316.

Sumner P, Nachev P, Vora N, Husain M, Kennard C (2004) Distinct cortical and collicular mechanisms of inhibition of return revealed with $\mathrm{S}$ cone stimuli. Curr Biol 14:2259-2263.

Thompson KG, Hanes DP, Bichot NP, Schall JD (1996) Perceptual and motor processing stages identified in the activity of macaque frontal eye field neurons during visual search. J Neurophysiol 76:4040-4055.

Toth LJ, Assad JA (2002) Dynamic coding of behaviourally relevant stimuli in parietal cortex. Nature 415:165-168.

Ungerleider JT, Mishkin M (1982) Two cortical visual systems. In: The analysis of visual behaviour (Ingle DJ, Mansfield RJW, Goodale MS, eds), pp 549-586. Cambridge, MA: MIT.

Vos JJ (1978) Colorimetric and photometric properties of a 2 degree fundamental observer. Color Res Appl 3:125-128.

White BJ, Kerzel D, Gegenfurtner KR (2006) Visually guided movements to color targets. Exp Brain Res 175:110-126. 